\title{
Health literacy: towards system level solutions
}

\author{
A new World Health Organization toolkit aimed at low and middle income countries could help \\ reduce health inequalities in the rest of the world too
}

\author{
Trisha Greenhalgh professor of primary care health sciences \\ Nuffield Department of Primary Care Health Sciences, University of Oxford, Oxford OX2 6GG, UK
}

In a new resource aimed at low and middle income countries, the World Health Organization has redefined health literacy as "the personal characteristics and social resources needed for individuals and communities to access, understand, appraise and use information and services to make decisions about health." 1

Low health literacy is associated with poor engagement with health services, health knowledge, concordance with prescribed medication, self management of illness, markers of disease progression, overall health status, and survival. It is also associated with high rates of hospital admission and use of emergency care. ${ }^{2-7}$

Low health literacy is more common in low income and minority ethnic groups, immigrants, people without full citizenship, those with fewer years of education, and older people; it is especially common in people who fall into several of these risk groups. ${ }^{8-11}$ People with low health literacy may feel ashamed and try to conceal it from professional carers and family members. ${ }^{12}$ Differences in health literacy explain a substantial proportion of inequity in the uptake and use of health services. ${ }^{11} 13$

Health literacy was traditionally conceptualised as an individual deficiency in verbal ability, numeracy, or both. It was measured as the equivalent school grade in classroom-style assessments of performance ${ }^{14}$ or as ability to read and interpret passages of health related text such as instructions on how to take medication. ${ }^{15}$ These widely used instruments have recently been challenged for being clinician centred, psychometrically questionable, and (at best) a partial measure of the things that influence patients' ability to stay healthy or manage their illnesses (especially when living in adverse physical and social environments and attempting to navigate a complex and unfamiliar healthcare system). ${ }^{16}{ }^{17}$ Two recent systematic reviews have added multiple dimensions to the construct of health literacy, including communication skills, motivation, confidence, trust, and the ability to access care. ${ }^{18} 19$

\section{Wider definitions}

A new generation of research into health literacy places assessment of individual literacy and numeracy within a broader public health model that emphasises the complex interdependencies between health understanding, health attitudes and behaviours, social determinants of health (such as income, education, material environment, and gender), and the design and delivery of health services. ${ }^{19}{ }^{20}$ Glass and McAtee talk of "risk regulators"--mediating structures (including such things as walkability of the local environment and accessible services) that link the social determinants of health with health behaviours and actions. ${ }^{21}$ Amartya Sen's health capabilities framework conceptualises health outcomes as the product of chances (the opportunities people have, which are economically, socially, and culturally determined) and the choices they make relative to these chances. ${ }^{22}$ People from educated and affluent backgrounds have better chances but also typically have socialised dispositions that orient them to making health positive choices.

Such framings imply that even when individual health literacy needs are identified, meeting these needs in particular subpopulations and risk groups requires a system-wide response. Accordingly, WHO is exhorting countries to assess and develop their own health literacy responsiveness - defined as "the way in which services, environments and products make health information and support available and accessible to people with different health literacy strengths and limitations."

Responsiveness tools take the perspective of a patient living in a particular set of real world circumstances and ask what is stopping him or her from achieving the best health outcomes in these circumstances. ${ }^{23}$ Patients may have advanced reading ability, for example, but they may not have the confidence or social skills to make their wishes clear in the clinical consultation.

In a pilot qualitative study, Buchbinder et al used the seed question "what abilities does a person with [your illness] need to have, in order to get, understand, and use health information to make informed decisions about their health?" with a similar seed question asked of health professionals. ${ }^{24}$ After several subsequent phases (both qualitative and quantitative), this team produced the multidimensional Health Literacy Questionnaire with robust psychometric properties. Its nine domains included "feeling understood and supported by healthcare providers," 
"social support for health," and "understanding health information well enough to know what to do." 25

Some domains on the questionnaire mainly reflect traits and capacities of the individual; others mainly reflect support from others and wider external factors. But in every domain, there are system level interventions that would help people with a low score achieve better health outcomes despite the low score. Policy makers who talk about "boosting" health literacy through educational programmes targeted at individuals ${ }^{26}$ may have failed to grasp this key point.

Community health literacy profiles could be used to target decisions on how to modify existing health systems in ways that reduce health inequalities, although their effectiveness is largely unproved. One such approach is the Ophelia (optimising health literacy and access to health information and services) pilot programme that began in Victoria, Australia. ${ }^{27}$ A locality based community partnership model is used to assess need (including testing defined populations on the nine domains of the Health Literacy Questionnaire); co-create acceptable and feasible solutions ("prioritising local wisdom, culture and systems"); and implement and evaluate against locally agreed metrics of success. ${ }^{28}$

The Ophelia programme, which is rooted in the principles of community based participatory research, ${ }^{29}$ is being delivered to deprived communities in diverse settings in South Africa and Thailand and to pilot sites in the UK, Denmark, and Indonesia. ${ }^{127}$ Its greatest strength is its flexibility to locally assessed need and use of codesign tools to develop solutions rather than seeking to impose a standardised "complex intervention." But although the model is reported to be popular, unqualified enthusiasm would be premature until evidence emerges from ongoing evaluations that its worthy goals are being met.

Competing interests: I have read and understood BMJ policy on declaration of interests and have no relevant interests to declare.

Provenance and peer review: Commissioned; not externally peer reviewed.

@trishgreenhalgh

1. World Health Organization. Health literacy toolkit for low- and middle-income countries. 2015. www.searo.who.int/entity/healthpromotion/documents/hl_tookit/en/:.

2 Bostock S, Steptoe A. Association between low functional health literacy and mortality in older adults: longitudinal cohort study. BMJ 2012;344:e1602.

3 Aboumatar HJ, Carson KA, Beach MC, Roter DL, Cooper LA. The impact of health literacy on desire for participation in healthcare, medical visit communication, and patient reported outcomes among patients with hypertension. J Gen Intern Med 2013;28:1469-76.

4 Al Sayah F, Majumdar SR, Williams B, Robertson S, Johnson JA. Health literacy and health outcomes in diabetes: a systematic review. J Gen Intern Med 2013;28:444-52.
5 DeWalt DA, Berkman ND, Sheridan S, Lohr KN, Pignone NP. Literacy and health outcomes. Journal J Gen Intern Med 2004:19:1228-39.

6 Lindquist LA, Go L, Fleisher J, Jain N, Friesema E, Baker DW. Relationship of health literacy to intentional and unintentional non-adherence of hospital discharge medications. $J$ Gen Intern Med 2012;27:173-8.

7 Nutbeam D. Health literacy as a public health goal: a challenge for contemporary health education and communication strategies into the 21 st century. Health Promotion Int 2000;15:259-67.

8 Gazmararian JA, Curran JW, Parker RM, Bernhardt JM, DeBuono BA. Public health literacy in America: an ethical imperative. Am J Prevent Med 2005;28:317-22.

9 Parker RM, Wolf MS, Kirsch I. Preparing for an epidemic of limited health literacy: Weathering the perfect storm. J Gen Intern Med 2008;23:1273-6.

10 Kreps GL, Sparks L. Meeting the health literacy needs of immigrant populations. Patient Educ Counsel 2008;71:328-32.

11 Howard DH, Sentell T, Gazmararian JA. Impact of health literacy on socioeconomic and racial differences in health in an elderly population. J Gen Intern Med 2006;21:857-61.

12 Parikh NS, Parker RM, Nurss JR, Baker DW, Williams MV. Shame and health literacy: the unspoken connection. Patient Educ Counsel 1996;27:33-9.

13 United Nations Economic and Social Council. Health literacy and the millennium development goals: United Nations Economic and Social Council (ECOSOC) regional meeting background paper (abstracted). J Health Commun 2010;15(suppl 2):211-23.

14 Davis TC, Long SW, Jackson RH, Mayeaux EJ, George RB, Murphy PW, Crouch MA. Rapid estimate of adult literacy in medicine: a shortened screening instrument. Fam Med 1993;25:391-5.

15 Parker RM, Baker DW, Williams MV. The test of functional health literacy in adults. J Gen Intern Med 1995:10:537-41.

16 Barber M, Buchbinder R, Osborne R, Elder C, Clerehan R, Busija L. Pronunciation of words is not a good measure of health literacy: determination of the validity of the Rapid Estimate of Adult Literacy in Medicine (REALM). Australian Rheumatology Association and Rheumatology Health Professional Association 48th annual scientific meeting, Perth, 20-24 May 2006.

17 Jordan JE, Osborne RH, Buchbinder R. Critical appraisal of health literacy indices revealed variable underlying constructs, narrow content and psychometric weaknesses. J Clin Epidemiol 2011;64:366-79.

18 Haun JN, Valerio MA, McCormack LA, Sørensen K, Paasche-Orlow. Health literacy measurement: an inventory and descriptive summary of 51 instruments. J Health Comm 2014;19(suppl 2):302-33.

19 Sørensen K, Van den Broucke S, Fullam J, Doyle G, Pelican J, Slonska Z, Brand H. Health literacy and public health: a systematic review and integration of definitions and models. BMC Pub Health 2012;12:80.

20 Sallis JF, Owen N, Fisher EB. Ecological models of health behavior. Health Behav Health Educ 2008;4:465-86.

21 Glass TA, McAtee MJ. Behavioral science at the crossroads in public health: extending horizons, envisioning the future. Soc Sci Med 2006:62:1650-71.

22 Weaver RR, Lemonde M, Payman N, Goodman WM. Health capabilities and diabetes self-management: the impact of economic, social, and cultural resources. Soc Sci Med 2014;102:58-68

23 Jordan JE, Buchbinder R, Osborne RH. Conceptualising health literacy from the patient perspective. Patient Educ Counsel 2010;79:36-42.

24 Buchbinder R, Batterham R, Ciciriello S, Newman S, Horgan B, Ueffing E, et al. Health literacy: what is it and why is it important to measure? J Rheumatol 2011:38:1791-7.

25 Osborne RH, Batterham RW, Elsworth GR, Hawkins M, Buchbinder R. The grounded psychometric development and initial validation of the Health Literacy Questionnaire (HLQ). BMC Pub Health 2013;13:658.

26 Koh HK, Berwick DM, Clancy CM, Baur C, Brach C, Harris LM, et al. New federal policy initiatives to boost health literacy can help the nation move beyond the cycle of costly "crisis care." Health Aff (Project Hope) 2012;31:434-43.

27 Batterham RW, Buchbinder R, Beauchamp A, Dodson S, Elsworth GR, Osborne RH. The OPtimising HEalth LlterAcy (Ophelia) process: study protocol for using health literacy profiling and community engagement to create and implement health reform. BMC Pub Health 2014;14:694.

28 World Health Organization. Health promotion glossary. Health Promot Int 1998;13:349-64

29 Jagosh J, Macaulay AC, Pluye P, Salsburg J, Bush PL, Henderson J, et al. Uncovering the benefits of participatory research: implications of a realist review for health research and practice. Milbank Q 2012;90:311-46.

Cite this as: BMJ 2015;350:h1026

๑ B BMJ Publishing Group Ltd 2015 